\title{
Mitochondrial transfer RNA mutations and hypertension
}

\author{
S.L. Yin, C. Lan, H. Pei, and Z.Q. Zhu \\ Department of Emergency Center, \\ The First Affiliated Hospital of Zhengzhou University, Zhengzhou, Henan, China \\ Corresponding author: S.L. Yin \\ E-mail: yinshlu@163.com
}

Genet. Mol. Res. 14 (4): 17692-17698 (2015)

Received August 14, 2015

Accepted October 7, 2015

Published December 21, 2015

DOI http://dx.doi.org/10.4238/2015.December.21.42

\begin{abstract}
Mutations in mitochondrial DNA have been found to be associated with hypertension. Of these, mitochondrial transfer RNA (mttRNA) is a hot spot for these pathogenic mutations. It is generally believed that these mutations may result in the failure of mt-tRNA metabolism, thereby worsening mitochondrial dysfunction and resulting in hypertension. mt-tRNA is known for its high frequency of polymorphisms and mutations, and the number of reports regarding mt-tRNA mutations and hypertension is increasing significantly. To better understand the molecular basis of maternally inherited hypertension, we reassessed the link between four mt-tRNA mutations (G15927A in tRNA ${ }^{\text {Thr }}$, C7492T in tRNA ${ }^{\text {Ser(UCN) }}$, A4386G in $\mathrm{tRNA}^{\mathrm{Gln}}$, and C14686T in tRNA ${ }^{\mathrm{Glu}}$ ) and hypertension. We first used the phylogenetic approach to investigate the deleterious roles of these mutations, then we used RNA Fold Web Server to predict the minimum free energy of these mt-tRNAs with and without mutations. Using the pathogenicity scoring system, we found that the G15927A and C7492T mutations are classified as pathogenic while all other studied mutations are neutral polymorphisms. Our study provides valuable information for the detection of pathogenic mt-tRNA mutations in hypertension.
\end{abstract}

Key words: Mitochondria; Mitochondrial tRNA mutations; Hypertension; Pathogenic mutations 


\section{INTRODUCTION}

Hypertension is a leading cause of stroke, heart disease and kidney failure. Despite significant advances in our understanding of the pathophysiology of hypertension, it remains one of the world's greatest public health issues (El Shamieh et al., 2012). It is estimated that one-third of the world's adult population will be hypertensive by 2025 (Kearney et al., 2005). Therefore, understanding the etiology of hypertension has become a major focus in research, with particular emphasis on the genetic basis of hypertension. Human hypertension is a condition associated with endothelial dysfunction and oxidative stress (Romero and Reckelhoff, 1999), and mitochondrial dysfunction has been implicated in both human and experimental hypertension (Bernal-Mizrachi et al., 2005). Early and recent investigations showed significant maternal familial aggregation of high blood pressure, which suggested a contribution of the mitochondrial genome to hypertension (Bengtsson et al., 1979; DeStefano et al., 2001; Yang et al., 2007). An inefficient metabolism caused by mitochondrial dysfunction in skeletal and vascular smooth muscle may lead to the elevation of systolic blood pressure and the development of hypertension (Wilson et al., 2004; Arrell et al., 2006). Moreover, maternal transmission of hypertension has been observed in some pedigrees, suggesting that mutations in mitochondrial DNA (mtDNA) are the molecular basis of this disorder (Schwartz et al., 2004). In particular, the A4295G and A4263G mutations in the mitochondrial transfer RNA lle (mt-tRNAlle) gene, and the $A 4401 G$ mutation in the junction between the mt-tRNA ${ }^{\mathrm{In}}$ and $m t-t R N A^{\text {Met }}$ genes are associated with hypertension (Li et al., 2008, 2009; Wang et al., 2011). These mt-tRNA mutations may result in transcriptional and translational defects, resulting in mitochondrial respiratory chain dysfunction; however, a poor genotype-to-phenotype association is common, as is the case of the mt-tRNA ${ }^{\text {met }}$ T4454C variant (Wang et al., 2014). Thus, determining pathogenic mutations and polymorphisms in mt-tRNA genes would be important for genetic counseling of patients with hypertension and their families.

In this study, we reassessed the association between four reported mt-tRNA mutations (G15927A in mt-tRNA ${ }^{\text {Thr }}$, C7492T in mt-tRNA ${ }^{\text {Ser(UCN) }}, A 4386 G$ in mt-tRNA ${ }^{\text {Gln }}$, and C14686T in mttRNA ${ }^{\text {Glu }}$ ) and hypertension (Liu et al., 2014). The G15927A mutation has already been shown to be pathogenic and associated with coronary heart disease, and the cybrid cell model containing this mutation shows a significant reduction in steady-state levels of mt-tRNA ${ }^{\text {Thr }}$ (Jia et al., 2013). Thus, in this study, we focused on the other three mt-tRNA mutations. To do this, we first carried out the evolutionary conservation analysis for these mt-tRNA mutations. Next, we used the RNA Fold Web Server to predict the minimum free energy (MFE) of mt-tRNAs with and without these mutations. We also used the pathogenicity scoring system to discern whether these mt-tRNA mutations contribute to the genetic susceptibility of hypertension.

\section{MATERIAL AND METHODS}

\section{Data extraction}

To begin our study, we took the clinical, genetic and molecular datasets from a recent paper concerning the association between mt-tRNA mutations and hypertension (Liu et al., 2014). For comparison, we searched in Pubmed Central and other public domains for studies examining the association between these mt-tRNA mutations and clinical disorders. 


\section{Determining the degree of evolutionary conservation}

With the purpose of understanding the role of mt-tRNA mutations in hypertension, we performed phylogenetic analysis for the conservation index $(\mathrm{Cl})$ of these mt-tRNA mutations. Briefly, 17 vertebrate mtDNA sequences were used for the inter-specific analysis. The $\mathrm{Cl}$ is then calculated by comparing the human nucleotide variants with the other 16 vertebrates. Of note, a $\mathrm{Cl}$ $\geq 70 \%$ is considered as having functional potential.

\section{Prediction of the secondary structure of mt-tRNA}

We used the RNA Fold Web Server (http://rna.tbi.univie.ac.at/cgi-bin/RNAfold.cgi) to predict the MFE secondary structure of mt-tRNAs with and without these mutations. In addition to MFE folding, equilibrium base-pairing probabilities were calculated via John McCaskill's partition function (PF) algorithm (McCaskill, 1990; Gruber et al., 2008).

\section{Pathogenicity scoring system for the mt-tRNA mutations}

The pathogenicity scoring system proposed by Yarham et al. (2011) was applied to evaluate the total score of the candidate pathogenic mt-tRNA mutations. According to this system, a variant is classified as "definitely pathogenic" with a score $\geq 11$ points, whereas a variant is defined as "possible pathogenic" with a score of 7-10 points and a "neutral polymorphism" has a score of 0-6 points.

\section{RESULTS}

\section{Molecular features of mt-tRNA mutations associated with hypertension}

Four mt-tRNA mutations have been found to be associated with hypertension. As shown in Figure 1, the G15927A (position 42) and C7492T (position 28) mutations are located in the

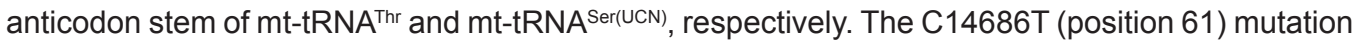
is located in the T-stem and the T4386C (position 15) mutation is located in the D-loop of the mttRNA $^{\text {Glu }}$ gene (Florentz et al., 2003). A careful review of the literature indicates that the T4386C mutation is implicated in coronary heart disease (Qin et al., 2014). Moreover, the T4386C mutation has been found in patients with mitochondrial myopathy (Ueki et al., 2006).

\section{Evolutionary conservation analysis of mt-tRNA mutations}

Conservation assessment of the bases in the mt-tRNA gene suggests their evolutionary importance; therefore, it is anticipated that mutations may have pathological consequences. In this study, we performed the analysis of the $\mathrm{Cl}$ of each mt-tRNA mutation using a phylogenetic approach. The Cls of C7492T, A4386G and C14686T are 73.1, 59.6 and 13.4\%, respectively. Only the C7492T mutation has a high $\mathrm{Cl}$, suggesting that it may be involved in the pathogenesis of hypertension.

\section{C7492T mutation alters mt-tRNA secondary structure}

To test whether nucleotide alterations affect the structure of mt-tRNA, the sequences of wild type and mutant tRNAs harboring the C7492T, A4386G and C14686T were predicted using 
the RNA Fold software. As displayed in Figure 2, except for the C7492T mutation, the mutations do not affect the secondary structure of the corresponding tRNAs, further indicating that the C7492T mutation may be involved in the pathogenesis of hypertension.

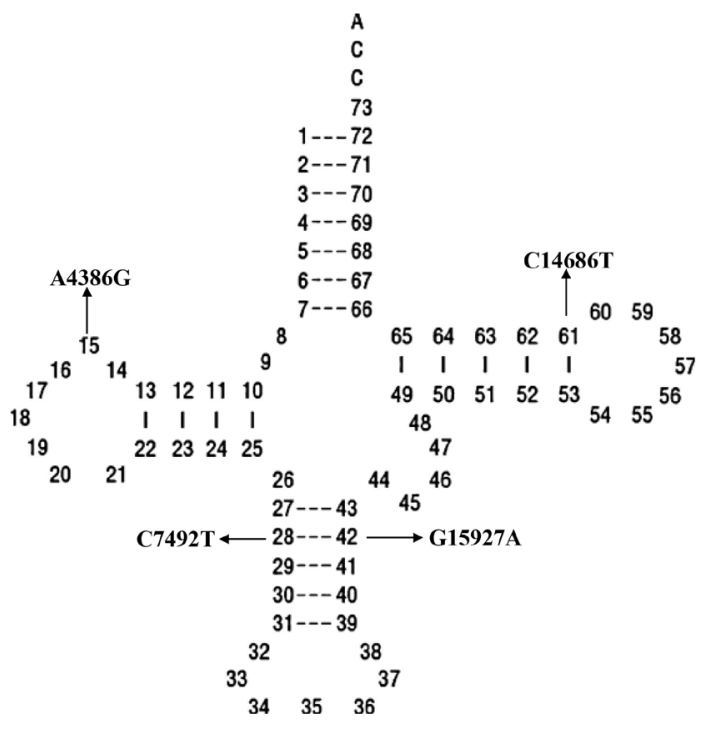

Figure 1. Cloverleaf structure of mt-tRNA with standard nucleotide numbering. The arrows indicate the positions of four mt-tRNA mutations: G15927A, C7492T, A4386G, and C14686T.

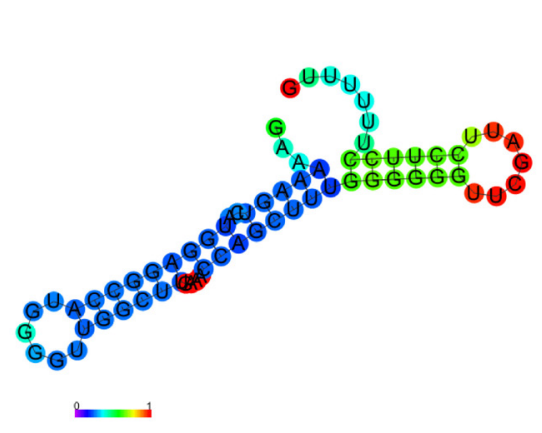

Wild type

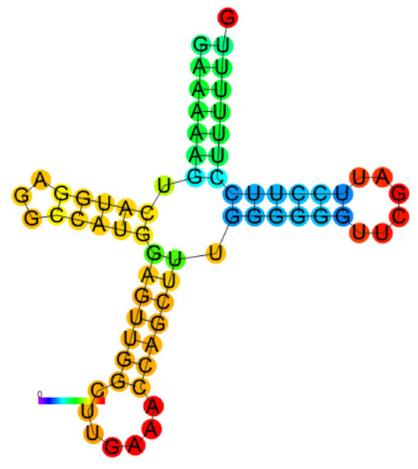

Mutant

Figure 2. Prediction of the secondary structure of mt-tRNA ${ }^{\text {Ser(UCN) }}$ with and without the C7492T mutation.

\section{Pathogenicity scoring system for the mt-tRNA mutations}

According to the revised pathogenicity scoring system for mt-tRNA mutations (Yarham et al., 2011), we classified the C7492T mutation as "possibly pathogenic" with a total score of 7 points (Table 1). The scores of the other mutations were: $\mathrm{A} 4386 \mathrm{G}=5$ points and $\mathrm{C} 14686 \mathrm{~T}=3$ points (data not shown). This indicates that they belong to the "neutral polymorphism" category. 
Table 1. Pathogenicity scoring system for the C7492T mutation.

\begin{tabular}{|c|c|c|c|}
\hline Scoring criteria & C7492T mutation & Score/20 & Classification \\
\hline More than one independent report & Yes & 2 & \\
\hline Evolutionary conservation of the base pair & One change & 2 & \\
\hline Variant heteroplasmy & No & 0 & \\
\hline Segregation of the mutation with disease & Yes & 2 & \\
\hline Histochemical evidence of mitochondrial disease & No evidence & 0 & \\
\hline Biochemical defect in complex I, III or IV & No & 0 & \\
\hline Evidence of mutation segregation with biochemical defect from single-fiber studies & No & 0 & \\
\hline Mutant mt-tRNA steady-state level or evidence of pathogenicity in trans-mitochondrial cybrid studies & Weak evidence & 1 & \\
\hline Total score & & 7 & Possibly pathogenic \\
\hline
\end{tabular}

Scoring system: $\leq 6$ points: neutral polymorphisms; $7-10$ points: possibly pathogenic; $\geq 11$ points: definitely pathogenic.

\section{DISCUSSION}

mt-tRNA point mutations are increasingly becoming recognized as important in disease, with novel pathogenic mutations being reported in all mt-tRNAs. Today, approximately 200 pathogenic mutations have been mapped to mt-tRNA genes (http://www.mitomap.org/MITOMAP), emphasizing the importance of mt-tRNA for mitochondrial function. It should be kept in mind that each cell contains hundreds to thousands of copies of mtDNA. Even in healthy individuals, a small population of polymorphic mutations is found in mtDNA. In fact, the Mitomap database includes a considerable amount of data on polymorphic mutations (McFarland et al., 2004). If a mutation in the mt-tRNA gene does not affect mtDNA replication or transcription, it may instead have a negative effect on the biogenesis and function of mt-tRNAs post-transcriptionally, whether it is in processing, post-transcriptional modification, aminoacylation or interactions with the mitoribosome during translation (Suzuki et al., 2011). Distinguishing the polymorphisms and mutations in mttRNAs is important and will lead to improved diagnosis and genetic counseling.

The current study investigates the possible link between four reported mt-tRNA mutations and hypertension. The G15927A mutation has already been identified as a pathogenic mutation, as it causes $\sim 80 \%$ reduction in the steady-state level of $\mathrm{mt}^{\mathrm{t}} \mathrm{tRNA} \mathrm{A}^{\mathrm{Th}}$. Moreover, in vivo mitochondrial protein labeling analysis showed $\sim 53 \%$ reduction in the rate of mitochondrial translation in mutant cells (Jia et al., 2013). The homoplasmic A4386G and C14686T mutations occur at positions that are not evolutionarily conserved and do not cause alterations in mt-tRNA secondary structure. In addition, the C14686T mutation occurs at position 61 in the T-loop of tRNA Glu and has not been reported in Mitomap or $\mathrm{mtDB}$, suggesting that it is a rare variant with allele frequencies less than $0.5 \%$. By contrast, the $\mathrm{C} 7492 \mathrm{~T}$ mutation is reported to increase the penetrance and expressivity of deafness associated with the 12S rRNA A1555G mutation (Lu et al., 2010). In the case of the C7492T mutation, phylogenetic conservation analysis supports that this mutation is conserved in different species and RNA Fold results show that it causes a change in the MFE of tRNA ${ }^{\text {Ser(UCN) }}$. This indicates that it may cause pathogenicity by disrupting the formation of the anticodon stem, which may in turn decrease the efficiency of codon-anticodon recognition and aminoacylation.

In summary, this is the first study to assess the association between the C7492T mutation and hypertension, and our data shows that this mutation is "possibly pathogenic" in hypertension. However, owing to the limitations of this study, further investigation using appreciate methods, such as a cybrid cell containing this mutation and determination of the mt-tRNA steady-state level, are required to evaluate the role of the C7492T mutation in hypertension. 


\section{Conflicts of interest}

The authors declare no conflict of interest.

\section{ACKNOWLEDGMENTS}

We thank the members of our department for discussion.

\section{REFERENCES}

Arrell DK, Elliott ST, Kane LA, Guo Y, et al. (2006). Proteomic analysis of pharmacological preconditioning: novel protein targets converge to mitochondrial metabolism pathways. Circ. Res. 99: 706-714.

Bengtsson B, Thulin T and Scherstén B (1979). Familial resemblance in casual blood pressure-a maternal effect? Clin. Sci. (Lond.) 57: 279s-281s.

Bernal-Mizrachi C, Gates AC, Weng S, Knutsen RH, et al. (2005). Vascular respiratory uncoupling increases blood pressure and atherosclerosis. Nature 435: 502-506.

DeStefano AL, Gavras H, Heard-Costa N, Bursztyn M, et al. (2001). Maternal component in the familial aggregation of hypertension. Clin. Genet. 60: 13-21.

El Shamieh S, Herbeth B, Azimi-Nezhad M, Orhan E, et al. (2012). Human formyl peptide receptor 1 C32T SNP interacts with age and is associated with blood pressure levels. Clin. Chim. Acta 413: 34-38.

Florentz C, Sohm B, Tryoen-Toth P, Pütz J, et al. (2003). Human mitochondrial tRNAs in health and disease. Cell. Mol. Life Sci. 60: 1356-1375.

Gruber AR, Lorenz R, Bernhart SH, Hofacker IL, et al. (2008). The Vienna RNA Website. Nucleic Acids Res. 1: W70-74.

Jia Z, Wang X, Qin Y, Xue L, et al. (2013). Coronary heart disease is associated with a mutation in mitochondrial tRNA. Hum. Mol. Genet. 22: 4064-4073.

Kearney PM, Whelton M, Reynolds K, Muntner P, et al. (2005). Global burden of hypertension: analysis of worldwide data. Lancet 365: 217-223.

Li R, Liu Y, Li Z, Yang L, et al. (2009). Failures in mitochondrial tRNAMet and tRNAGIn metabolism caused by the novel 4401A4G mutation are involved in essential hypertension in a Han Chinese Family. Hypertension 54: 329-337.

Li Z, Liu Y, Yang L, Wang S, et al. (2008). Maternally inherited hypertension is associated with the mitochondrial tRNAlle A4295G mutation in a Chinese family. Biochem. Biophys. Res. Commun. 367: 906-911.

Liu Y, Zhu Q, Zhu C, Wang X, et al. (2014). Systematic analysis of the clinical and biochemical characteristics of maternally inherited hypertension in Chinese Han families associated with mitochondrial. BMC Med. Genomics 7: 73.

Lu J, Qian Y, Li Z, Yang A, et al. (2010). Mitochondrial haplotypes may modulate the phenotypic manifestation of the deafnessassociated 12S rRNA 1555A>G mutation. Mitochondrion 10: 69-81.

McCaskill JS (1990). The equilibrium partition function and base pair binding probabilities for RNA secondary structure. Biopolymers 29: 1105-1119.

McFarland R, Elson JL, Taylor RW, Howell N, et al. (2004). Assigning pathogenicity to mitochondrial tRNA mutations: when "definitely maybe" is not good enough. Trends Genet. 20: 591-596.

Qin Y, Xue L, Jiang P, Xu M, et al. (2014). Mitochondrial tRNA variants in Chinese subjects with coronary heart disease. J. Am. Heart Assoc. 3: e000437.

Romero JC and Reckelhoff JF (1999). State-of-the-art lecture. Role of angiotensin and oxidative stress in essential hypertension. Hypertension 34: 943-949.

Schwartz F, Duka A, Sun F, Cui J, et al. (2004). Mitochondrial genome mutations in hypertensive individuals. Am. J. Hypertens. 17: 629-635.

Suzuki T, Nagao T and Suzuki T (2011). Human mitochondrial diseases caused by lack of taurine modification in mitochondrial tRNAs. Wiley. Interdiscip. Rev.: RNA 2: 376-386.

Ueki I, Koga Y, Povalko N, Akita Y, et al. (2006). Mitochondrial tRNA gene mutations in patients having mitochondrial disease with lactic acidosis. Mitochondrion 6: 29-36.

Wang S, Li R, Fettermann A, Li Z, et al. (2011). Maternally inherited essential hypertension is associated with the novel $4263 A>G$ mutation in the mitochondrial tRNAlle gene in a large Han Chinese family. Circ. Res. 108: 862-870.

Wang Y, Dong P, Li L, Li X, et al. (2014). The mitochondrial tRNA(Met) 4454T > C variant may not be associated with essential hypertension in Han Chinese population. Mitochondrial DNA 25: 124-125. 
Wilson FH, Hariri A, Farhi A, Zhao H, et al. (2004). A cluster of metabolic defects caused by mutation in a mitochondrial tRNA. Science 306: 1190-1194.

Yang Q, Kim SK, Sun F, Cui J, et al. (2007). Maternal influence on blood pressure suggests involvement of mitochondrial DNA in the pathogenesis of hypertension: the Framingham Heart Study. J. Hypertens. 25: 2067-2073.

Yarham JW, Al-Dosary M, Blakely EL, Alston CL, et al. (2011). A comparative analysis approach to determining the pathogenicity of mitochondrial tRNA mutations. Hum. Mutat. 32: 1319-1325. 